\title{
Longitudinal Development of Brain Iron Is Linked to Cognition in Youth
}

\author{
[DBart Larsen, ${ }^{1}$ Josiane Bourque, ${ }^{1}$ Tyler M. Moore, ${ }^{1}$ Azeez Adebimpe, ${ }^{1}$ Monica E. Calkins, ${ }^{1}$ Mark A. Elliott, ${ }^{1,2}$ \\ Ruben C. Gur, ${ }^{1,2}$ Raquel E. Gur, ${ }^{1,2}$ Paul J. Moberg, ${ }^{1}$ David R. Roalf, ${ }^{1}$ Kosha Ruparel, ${ }^{1}$ Bruce I. Turetsky, ${ }^{1}$

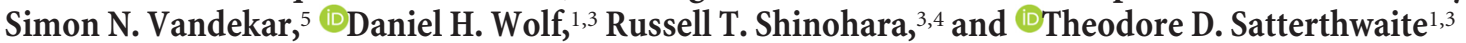 \\ ${ }^{1}$ Department of Psychiatry, Perelman School of Medicine, ${ }^{2}$ Department of Radiology, ${ }^{3}$ Center for Biomedical Image Computing and Analytics, ${ }^{4}$ Department \\ of Biostatistics, Epidemiology and Informatics, Pennsylvania Statistics in Imaging and Visualization Center, University of Pennsylvania, Philadelphia, \\ Pennsylvania 19104, and ${ }^{5}$ Department of Biostatistics, Vanderbilt University, Nashville, Tennessee 37235.
}

Brain iron is vital to multiple aspects of brain function, including oxidative metabolism, myelination, and neurotransmitter synthesis. Atypical iron concentration in the basal ganglia is associated with neurodegenerative disorders in aging and cognitive deficits. However, the normative development of brain iron concentration in adolescence and its relationship to cognition are less well understood. Here, we address this gap in a longitudinal sample of 922 humans aged 8-26 years at the first visit $(\mathrm{M}=15.1, \mathrm{SD}=3.72 ; 336$ males, 486 females) with up to four multiecho $\mathrm{T} 2^{\star}$ scans each. Using this sample of 1236 imaging sessions, we assessed the longitudinal developmental trajectories of tissue iron in the basal ganglia. We quantified tissue iron concentration using R2* relaxometry within four basal ganglia regions, including the caudate, putamen, nucleus accumbens, and globus pallidus. The longitudinal development of R2* was modeled using generalized additive mixed models (GAMMs) with splines to capture linear and nonlinear developmental processes. We observed significant increases in $\mathrm{R} 2^{\star}$ across all regions, with the greatest and most prolonged increases occurring in the globus pallidus and putamen. Further, we found that the developmental trajectory of $\mathrm{R} 2 *$ in the putamen is significantly related to individual differences in cognitive ability, such that greater cognitive ability is increasingly associated with greater iron concentration through late adolescence and young-adulthood. Together, our results suggest a prolonged period of basal ganglia iron enrichment that extends into the midtwenties, with diminished iron concentration associated with poorer cognitive ability during late adolescence.

Key words: adolescence; basal ganglia; cognition; development; iron; R2*

Significance Statement

Brain tissue iron is essential to healthy brain function. Atypical basal ganglia tissue iron levels have been linked to impaired cognition in iron deficient children and adults with neurodegenerative disorders. However, the normative developmental trajectory of basal ganglia iron concentration during adolescence and its association with cognition are less well understood. In the largest study of tissue iron development yet reported, we characterize the developmental trajectory of tissue iron concentration across the basal ganglia during adolescence and provide evidence that diminished iron content is associated with poorer cognitive performance even in healthy youth. These results highlight the transition from adolescence to adulthood as a period of dynamic maturation of tissue iron concentration in the basal ganglia.

\section{Introduction}

Brain tissue iron is essential to healthy brain function. Distinct from heme-iron, tissue iron binds to the intracellular protein

\footnotetext{
Received Oct. 9, 2019; revised Dec. 19, 2019; accepted Jan. 4, 2020.

Author contributions: B.L., R.E.G., R.T.S., and T.D.S. designed research; B.L., M.E.C., P.J.M., D.R.R., K.R., and B.I.T. performed research; B.L., J.B., T.M.M., and A.A. analyzed data; B.L. wrote the first draft of the paper; B.L., J.B., T.M.M., M.A.E., R.C.G., R.E.G., P.J.M., D.R.R., K.R., B.I.T., S.N.V., D.H.W., R.T.S., and T.D.S. edited the paper; B.L. and T.D.S. wrote the paper; J.B., A.A., M.A.E., R.C.G., and S.N.V. contributed unpublished reagents/analytic tools.

This work was supported by the National Institutes of Health (T32MH019112 to B.L., T32MH014654 to B.L., R01MH119219 to R.E.G. \& R.C.G., R01MH117014 to R.C.G., U01-MH081902 to R.E.G., MH063381 to P.J.M., MH108895 to P.J.M., R01MH099156 to B.I.T., R01MH113565 to D.H.W., K01MH102609 to D.R.R., R01MH112847 to
}

ferritin and is stored in neurons, oligodendrocytes, astrocytes, and microglia (Todorich et al., 2009; Mills et al., 2010; Ward et al., 2014). Within these cells, iron is required as a cofactor for multiple fundamental neural processes. As part of the process of cellular respiration, iron is necessary for ATP synthesis (Ward et al.,

\footnotetext{
R.T.S. \& T.D.S., R01MH113550 to T.D.S., R01MH120482 to T.D.S., R01MH107703 to T.D.S.); Canadian Institutes of Health Research (CIHR396349 to J.B.)

The authors declare no competing financial interests.

Correspondence should be addressed to Bart Larsen at bart.larsen@pennmedicine.upenn.edu.

https://doi.org/10.1523/JNEUROSCI.2434-19.2020

Copyright $\odot 2020$ the authors
} 
2014; Paul et al., 2017). In oligodendrocytes, iron supports the high metabolic demand of myelin maintenance and synthesis and functions as a cofactor for lipid synthesis (Connor and Menzies, 1996; Todorich et al., 2009; Möller et al., 2019). Iron is also a necessary cofactor for tyrosine hydroxylase (Ramsey et al., 1996) and monoamine oxidase (Youdim et al., 1976; Lu et al., 2017; Youdim, 2018), enzymes essential for dopamine synthesis, as well as tryptophan hydroxylase (Kuhn et al., 1980), which is necessary for serotonin synthesis. Tissue iron is heterogeneously distributed in the brain, with the greatest iron concentration in basal ganglia structures such as the caudate, putamen, nucleus accumbens, and globus pallidus (Hallgren and Sourander, 1958; Thomas et al., 1993; Brass et al., 2006). The reason for pronounced iron enrichment of the basal ganglia and the mechanism by which it occurs are not fully understood, but the basal ganglia are rich in dopamine and play a critical role in a multitude of cognitive and motor functions as part of the corticobasal ganglia-thalamocortical loops (Haber and Knutson, 2010).

Brain tissue iron content is homeostatically regulated (Moos, 2002; Burdo and Connor, 2003), and atypical iron concentration in the basal ganglia is associated with neurological disorders and impaired cognitive function. Abnormally high brain iron content in aging is associated with Parkinson's disease (Lv et al., 2011; Zucca et al., 2017), Alzheimer's disease (Griffiths and Crossman, 1993; Bartzokis et al., 2000), and Huntington's disease (Bartzokis et al., 1999; Bartzokis and Tishler, 2000; Kell, 2010), as well as cognitive decline (Pujol et al., 1992; Penke et al., 2012; Adamo et al., 2014; Daugherty and Raz, 2015; Ghadery et al., 2015). In contrast, iron deficiency in early development (e.g., gestation through childhood) leads to atypically low brain tissue iron concentration (Erikson et al., 2000, 2001; Beard, 2003) and is similarly associated with abnormal basal ganglia dopaminergic neurobiology and neurochemistry (Erikson et al., 2001; Lozoff and Georgieff, 2006; Jellen et al., 2013), and cognitive impairment (Grantham-McGregor and Ani, 2001; Halterman et al., 2001; McCann and Ames, 2007; Shafir et al., 2008; Carter et al., 2010; Lozoff, 2011). While substantial work has been done to delineate these critical links between atypical brain tissue iron concentration and neurocognitive function in early development and late-life, less is known about how tissue iron concentration normatively develops during adolescence, and whether normative variation in the developmental trajectory of tissue iron concentration is related to cognitive ability.

Here, we address this question by assessing the development of tissue iron concentration in a normative sample of 922 adolescents and young adults (aged 8-26y) with up to four longitudinal measurements per participant, resulting in 1236 total scans after strict quality assessment. In this sample, we assess iron content using $\mathrm{R} 2^{*}$ relaxation $\left(1 / \mathrm{T} 2^{*}\right)$, which is linearly related to postmortem tissue iron concentration (Langkammer et al., 2010; Sedlacik et al., 2014). We used generalized additive mixed models to characterize the normative developmental trajectory of tissue iron concentration across the basal ganglia and to assess whether these normative trajectories vary with individual differences in multiple domains of cognitive ability. As described below, we find that tissue iron concentration continues to increase throughout adolescence and into young adulthood, with the greatest increases occurring in the putamen and globus pallidus. Further, we find that individual differences in cognitive performance are associated with the developmental enrichment of iron concentration in the putamen.
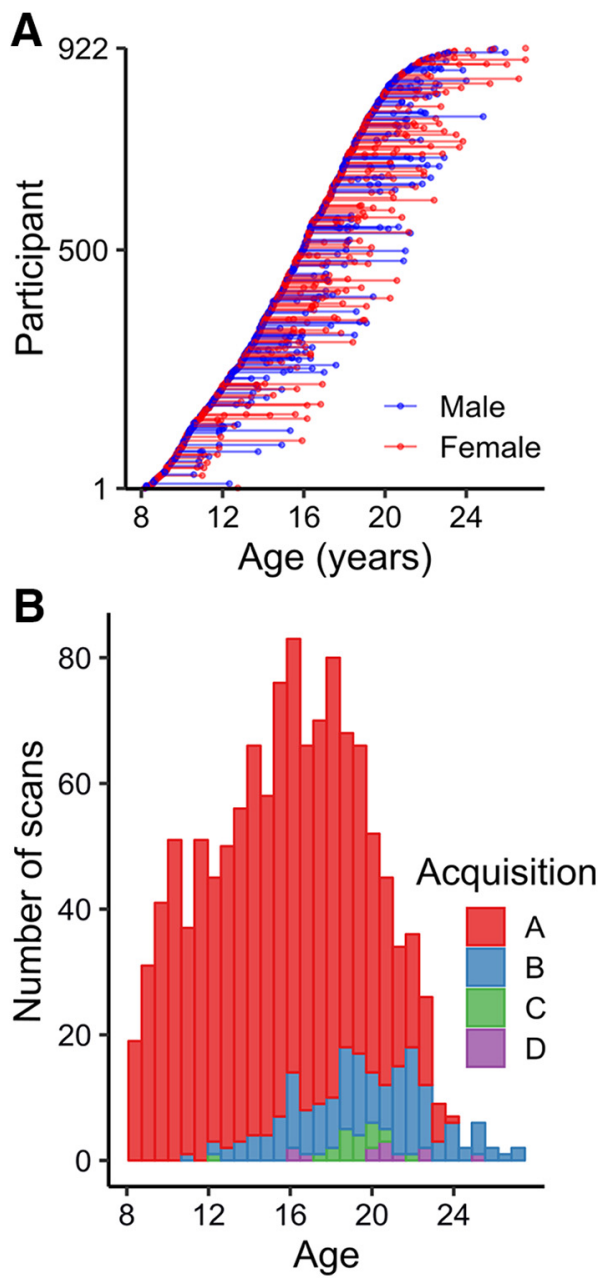

Figure 1. Final neuroimaging sample characteristics. A, Final imaging sample-after exclusions - was composed of 922 individuals and 1236 total scans. Each point depicts an individual scan, with lines connecting individual participants. Participant sex is indicated as red (female) or blue (male). $\boldsymbol{B}$, Stacked histogram of acquisitions used for scans across the age range (Table 1).

\section{Materials and Methods}

Participants. Neuroimaging data were obtained from a communitybased sample of 1543 youth that were part of the Philadelphia Neurodevelopmental Cohort and related follow-up studies (Jirsaraie et al., 2019; Roalf et al., 2019) (ages 8.17-26.92 at first visit, $\mathrm{M}=15.19, \mathrm{SD}=3.75$; male/female $=728 / 819)$. This neuroimaging dataset included up to five longitudinal visits per participant, resulting in 2321 total scans. From this original sample, 309 participants (419 scans) were excluded based on health criteria, including psychoactive medication use at the time of study, medical problems that could impact brain function, a history of psychiatric hospitalization, and gross structural brain abnormalities. A total of 641 scans were excluded for poor $\mathrm{T} 2{ }^{*}$ image quality, including head motion, hyperintensities, and susceptibility artifacts, which was assessed with manual quality assurance procedures (see below). In sum, following health exclusions and rigorous quality assurance we retained 922 participants (ages 8.17-26.92 at first visit, $\mathrm{M}=15.1$, $\mathrm{SD}=3.72$; male/female $=436 / 486$ ) with up to four longitudinal neuroimaging visits per participant, resulting in 1236 total scans (Fig. 1). From this neuroimaging sample, cognitive performance data (described below) was available for 818 individuals, with up to three time points per individual (ages 8.17-26.92 at first visit, $\mathrm{M}=14.84, \mathrm{SD}=3.57$; male/female = 389/429), resulting in 1086 total cognitive assessments.

Structural image processing. A high-resolution T1-weighted image was acquired for all visits for the purpose of nonlinear registration to a study specific template. The study-specific template was generated from a sam- 
Table 1. Sequence counts and descriptions

\begin{tabular}{|c|c|c|c|c|c|c|c|c|}
\hline Sequence & $\begin{array}{l}\text { No. of } \\
\text { scans }\end{array}$ & $\begin{array}{l}\text { Scanner } \\
\text { model }\end{array}$ & TR & TE & $\Delta \mathrm{TE}$ & Voxel dimensions (mm) & FOV & $\begin{array}{l}\text { Flip } \\
\text { angle }\end{array}$ \\
\hline$A^{*}$ & 1043 & Trio & 1000 & $2.69,5.27$ & 2.58 & $3.75 \times 3.75 \times 4$ & $240 \times 240$ & $60^{\circ}$ \\
\hline B & 161 & Trio & 1000 & $3.13,5.59$ & 2.46 & $3.75 \times 3.75 \times 4$ & $240 \times 240$ & $60^{\circ}$ \\
\hline$C^{*}$ & 20 & Trio & 1000 & $2.69,5.27$ & 2.58 & $3.75 \times 3.75 \times 4$ & $240 \times 240$ & $60^{\circ}$ \\
\hline D & 12 & Trio & 1050 & $4.06,6.52$ & 2.46 & $3.75 \times 3.75 \times 4$ & $240 \times 240$ & $60^{\circ}$ \\
\hline
\end{tabular}

*Sequences $\mathrm{A}$ and $\mathrm{C}$ differed in that sequence $\mathrm{A}$ used an advanced shimming procedure.

ple of 120 PNC subjects balanced across sex, race, and age bins using the "buildtemplateparallel" procedure in ANTs (Avants et al., 2011a). Each participant's high-resolution structural image was processed using Advanced Normalization Tools (ANTs) software and the structural processing pipeline included bias field correction (Tustison et al., 2010), brain extraction using a hybrid registration/segmentation approach (Avants et al., 2011b), and diffeomorphic registration to the studyspecific template using SyN deformation provided by ANTs (Avants et al., 2008; Klein et al., 2009).

$R 2^{*}$ acquisition and preprocessing. $\mathrm{T} 2{ }^{\star}$ data were estimated from multiecho gradient echo (mGRE) images acquired for each participant. Sequence parameters are summarized in Table 1. It should be noted that a subset of the data was acquired with minor variations in acquisition parameters. However, all scans were acquired on the same scanner, $99 \%$ used the same repetition time (TR, $1000 \mathrm{~ms}$ ), and $86 \%$ used the same echo spacing ( $\triangle T E, 2.58 \mathrm{~ms}$ ). Notably, for the minority of participants for whom $\triangle T E$ varied, the difference between acquisitions was only 0.12 $\mathrm{ms}$. As a conservative step, to ensure that the variability in $\mathrm{T} 2^{*}$ acquisition parameters did not affect our results, $\mathrm{T}^{*}$ data were harmonized across acquisitions before statistical analysis using ComBat (Johnson et al., 2007) (see below).

Acquired mGRE images were first smoothed with a $3 \mathrm{~mm}$ FWHM kernel. $\mathrm{T} 2{ }^{\star}$ was calculated from the pixel intensity of the two echo images according to the following:

$$
T 2^{\star}=\frac{-\Delta T E}{\operatorname{In}\left(\frac{I_{T E 2}}{I_{T E 1}}\right)}
$$

where $I_{\mathrm{TE} 1}$ and $I_{\mathrm{TE} 2}$ refer to the signal intensity at the first and second echo. $\mathrm{T} 2{ }^{*}$ images were brain extracted using coregistration to the brainextracted structural image (above). $\mathrm{T} 2 *$ images were finally spatially normalized to the study-specific template by concatenating the affine registration between the $\mathrm{T} 2{ }^{*}$ image and structural image and the nonlinear registration of the structural image to the study template. $\mathrm{R} 2^{*}$ was calculated as $\left(\frac{1}{T 2^{\star}}\right)$.

$R 2^{\star}$ quality assurance. All R2* images were visually inspected by BL for data quality and the presence of artifacts, including motion artifacts, susceptibility artifacts, extreme values, and $\mathrm{T} 2{ }^{\star}$ hyperintensities, any of which could impact the $\mathrm{R}^{*}$ signal in the basal ganglia. Visual inspection was performed blind to all experimental variables. Each image was rated as "pass" or "fail," and only images that passed inspection were included in statistical analyses. This procedure assured that only high-quality scans would be included in analyses (Fig. 2).

Region of interest identification. We evaluated basal ganglia regions of interest as in prior work (Langkammer et al., 2010; Sedlacik et al., 2014; Daugherty and Raz, 2015), including the caudate, putamen, nucleus accumbens, and globus pallidus. These areas were identified in template space by a segmentation of the structural template brain using ANTs joint label fusion (Wang et al., 2013). Regional R2* values were calculated as the mean across all voxels in each region across both hemispheres. As the regional values were extracted from spatially normalized data, the number of voxels did not differ across participants.

Cognitive assessment. The Penn computerized neurocognitive battery (CNB) was administered to 818 participants with up to three time points per participant (ages 8.17-26.92 at first visit, $\mathrm{M}=14.84, \mathrm{SD}=3.57$; male/female $=389 / 429$ ), resulting in 1067 total cognitive assessments.

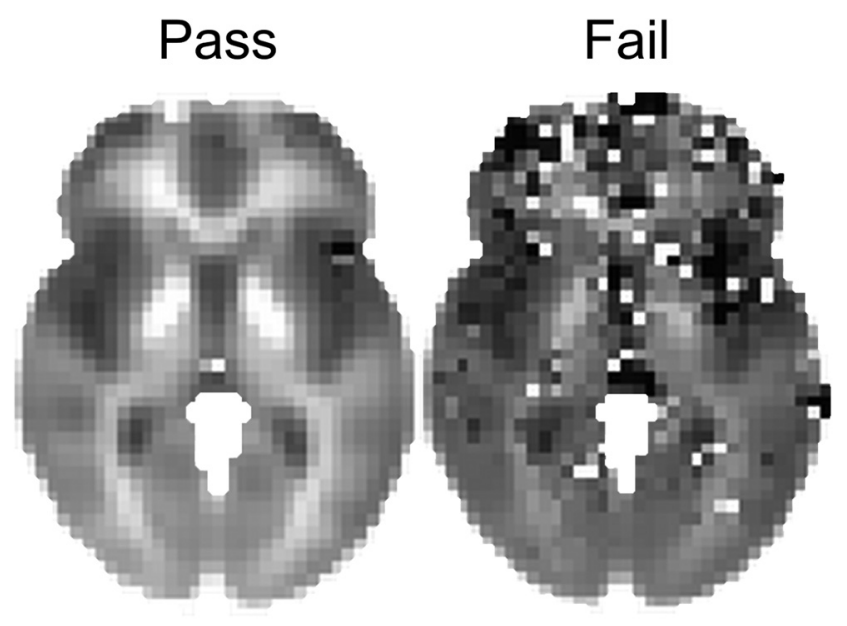

Figure 2. Outcome of quality assurance procedures. The voxelwise mean of all $R 2 *$ scans that passed quality assurance $(n=1236)$ is depicted on the left, and the voxelwise mean of all $\mathrm{R} 2 *$ scans that failed $(n=641)$ is depicted on the right.

The CNB consists of 14 tests adapted from tasks applied in functional neuroimaging to evaluate a broad range of cognitive domains (Gur et al., 2010, 2012). These domains include executive control (abstraction and mental flexibility, attention, working memory), complex cognition (verbal reasoning, nonverbal reasoning, spatial processing), episodic memory (verbal, facial, spatial), social cognition (emotion identification, emotion intensity differentiation, age differentiation), and motor speed. Accuracy and speed for each task were $z$-transformed and combined into an efficiency variable. Factor scores were calculated separately for an overall factor and four correlated subfactors. For the overall factor, tests were modeled in a unidimensional (one-factor) model with least-squares extraction. The subfactor scores (complex cognition, executive function, social cognition, and memory) were calculated using an exploratory factor analysis (EFA) with least-squares extraction and oblique geomin rotation. The dimensionality was determined theoretically based on the design of the CNB and prior work (Moore et al., 2015; Gur and Gur, 2016). Note that unidimensional models were used for the overall scores despite multidimensionality because the ratio of first to second eigenvalues for efficiency were all $>3.0$, providing some support for use of a unidimensional model (Reise et al., 2015).

Group-level statistical analysis. Before all statistical analyses, R2* data were harmonized to control for subtle differences in $\mathrm{T} 2^{*}$ acquisition parameters using ComBat, a batch-effect correction tool developed for genomics data (Johnson et al., 2007) and previously applied to harmonize multisite neuroimaging data, including in developmental studies (Fortin et al., 2017, 2018). ComBat removes unwanted interacquisition variability while preserving variability in biological variables of interest. ComBat was performed separately for observations included in developmental models (including covariates for age, sex, and visit number) and cognitive models (including covariates for age, sex, visit number, and cognitive performance).

We first tested for mean level differences among our basal ganglia regions of interest. These region of interest comparisons were performed as post hoc contrasts of estimated marginal means from a linear mixed effects model that included covariates for age, sex, and visit number. 
Significance values were adjusted using the Bonferroni correction for multiple comparisons.

To assess developmental trajectories of $\mathrm{R} 2^{*}$, we used splines within a generalized additive mixed model (GAMM) to flexibly capture linear or nonlinear age effects and account for longitudinal repeated measures. For each region of interest, we first tested for a significant age-by-sex interaction (i.e., factor-smooth interaction). If the interaction was not significant, the GAMM was refit removing the interaction term and modeling sex as an additive covariate. To test for windows of significant change across the age range, we calculated the first derivative of the smooth function of age from the GAMM model using finite differences, and then generated a simultaneous $95 \%$ confidence interval of the derivative (Simpson, 2018) using the gratia package in R. Intervals of significant change were identified as areas where the simultaneous confidence interval of the derivative does not include zero.

We next investigated the relationship between cognition and $\mathrm{R} 2^{*}$ and whether developmental trajectories of R2* interacted with cognitive ability. To test for a main effect of overall cognitive performance, we fit a GAMM with additive smooth terms for cognition and age and a main effect of sex. To assess whether developmental trajectories of $\mathrm{R} 2^{*}$ varied by cognitive ability, we allowed the smoothed age effect in the GAMM to interact with cognitive performance. All GAMMs were fit in R using the 'gamm' function from the $m g c v$ package (Wood, 2004, 2017). To model this continuous interaction, we compared two interaction models within the GAMM framework: a fully nonlinear interaction (bivariate smooth) and a nonlinear-by-linear interaction in which the smooth effect of age interacts with a linear effect of cognitive performance (varying coefficient model). Model selection was performed using Bayesian information criterion (BIC), and the significance of the interaction term was evaluated for the selected model. Finally, we evaluated three-way age-bycognition-by-sex interactions, which were not significant and not considered further.

All models included visit number as a covariate factor of no interest and participant ID as a random effect. For all GAMMs, significance of the fixed effect smooth terms was assessed in a fixed degree-of-freedom context to ensure stable and accurate estimation, and all $p$-values of interest were confirmed using a parametric bootstrap likelihood ratio test ( $p b$ krtest package) for significance estimation in the mixed model context (Halekoh and Højsgaard, 2014). Multiple-comparison adjustment was performed with Bonferroni correction.

Code accessibility. Custom $\mathrm{R}$ code for all primary statistical analyses as well as example bash code for R2* calculation is available at https:// github.com/PennBBL/Larsen_IronDevelopment.

\section{Results}

\section{Regional differences in iron concentration}

Model estimated regional $\mathrm{R}^{\star}$ values-covarying for sex and visit number-closely matched prior studies (Hallgren and Sourander, 1958; Bartzokis et al., 1997, 2007; Aquino et al., 2009; Péran et al., 2009; Haacke et al., 2010 p.201; Langkammer et al., 2010; Sedlacik et al., 2014; Ghadery et al., 2015; Hect et al., 2018). Globus pallidus had the greatest model estimated marginal mean $(\mathrm{EMM}) \mathrm{R} 2 *(\mathrm{EMM}=22.33$, confidence interval $\{\mathrm{CI}\}=[22.22$, $22.44])$, followed by nucleus accumbens $(\mathrm{EMM}=17.59, \mathrm{CI}=$ $[17.48,17.69])$, putamen $(\mathrm{EMM}=16.96, \mathrm{CI}=[16.85,17.07])$, and caudate $(\mathrm{EMM}=15.17, \mathrm{CI}=[15.06,15.28])$ (Fig. 3). All regions significantly differed from each other (Table 2 ).

\section{Age-related increases in R2 ${ }^{\star}$ across the basal ganglia}

$\mathrm{R} 2^{\star}$ significantly increased with age across all basal ganglia regions of interest while covarying for sex and visit number (Fig. 4, Table 3). There was a significant interaction with sex in the globus pallidus such that $\mathrm{R} 2{ }^{*}$ began to reach plateau at age $22.2 \mathrm{y}$ in females and continued to increase in males. Females tended to have greater $\mathrm{R} 2^{\star}$ regardless of age in the nucleus accumbens, though the effect did not survive at the Bonferroni adjusted $\alpha$ of $0.0125(t=2.26, p=0.024)$. Analysis of the derivatives of the

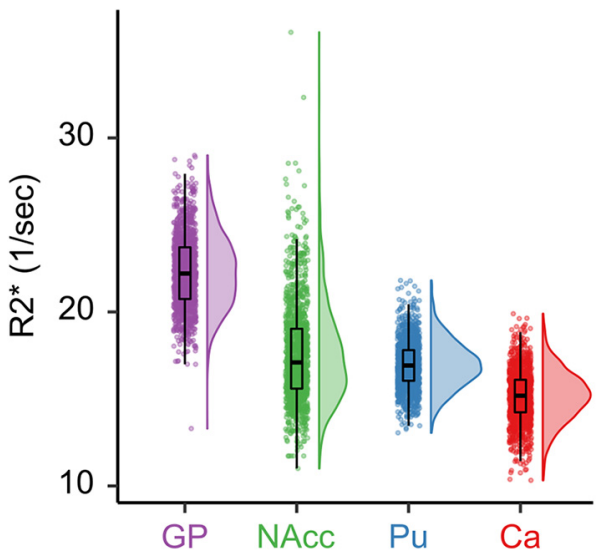

Figure 3. Sample distribution of $\mathrm{R} 2^{*}$ across basal ganglia regions of interest. Individual points represent regional $R 2{ }^{*}$ values for individual scans. Boxplots indicate the median value, as well as the first and third quartiles of the sample distribution. GP, Globus pallidus; NAcc, nucleus accumbens; Pu, putamen; Ca, caudate.

Table 2. Model estimated differences in R2* across the basal ganglia controlling for age, sex, and visit

\begin{tabular}{llllll}
\hline Contrast & Difference & $z$ & $p_{\text {Bonf }}$ & $95 \% \mathrm{Cl}_{\text {Low }}$ & $95 \% \mathrm{Cl}_{\text {High }}$ \\
\hline Caudate - Putamen & -1.79 & -26.36 & $<0.001$ & -1.96 & -1.61 \\
Caudate - Nucleus Accumbens & -2.41 & -34.63 & $<0.001$ & -2.59 & -2.23 \\
Caudate - Pallidum & -7.16 & -102.65 & $<0.001$ & -7.34 & -6.98 \\
Putamen - Nucleus Accumbens & -0.63 & -8.28 & $<0.001$ & -0.81 & -0.45 \\
Putamen - Pallidum & -5.37 & -76.29 & $<0.001$ & -5.55 & -5.19 \\
Nucleus Accumbens - Pallidum & -4.75 & -68.02 & $<0.001$ & -4.92 & -4.57 \\
\hline
\end{tabular}

fitted age trajectories identified that $\mathrm{R} 2^{*}$ significantly increased between ages $8.2-17.3$ years in nucleus accumbens, 10.2-26.9 years in the putamen, ages $8.2-16.1$ years in the caudate, and 8.2-25.9 years for males and 8.2-22.2 years for females in the globus pallidus (Fig. 4).

\section{Developmental trajectories of $\mathrm{R}^{\star}{ }^{\star}$ are related to cognitive performance}

We next investigated whether the developmental trajectories we observed varied with of cognitive ability. We observed that the developmental trajectory of $\mathrm{R}^{*}$ in the putamen significantly interacted with overall cognitive performance at a Bonferroniadjusted $\alpha$ of .0125 (s(Age):Overall $F=3.67, p=0.006$; Fig. 5). This developmental interaction was such that the association between cognitive ability and $\mathrm{R} 2^{\star}$ emerged during adolescence, with poorer performance being increasingly associated with diminished $\mathrm{R} 2^{\star}$ levels. To understand the specific cognitive domains that contributed to this effect, we conducted specificity analyses focusing on four cognitive domains: complex cognition, social cognition, executive function, and memory. Cognitive factor loadings and correlations can be found in Table 4. We found that developmental trajectories of $\mathrm{R} 2{ }^{*}$ most strongly interacted with complex cognition performance (s(Age):Complex $F=3.94$, $p=0.004$; Fig. $6 A$ ). A similar pattern was observed for social cognition (s(Age):Social $F=2.67, p=0.031$; Fig. $6 B$ ) and executive function (s(Age): Executive $F=2.66, p=0.032$; Fig. $6 C$ ), though the effects were not significant at a Bonferroni-adjusted $\alpha$ of .0125. The effect was not significant for memory performance (s(Age):Memory $F=1.03, p=0.39$; Fig. $6 D$ ).

\section{Discussion}

Here, we capitalized upon a large sample of youth with longitudinal neuroimaging data to characterize the developmental tra- 
jectories of tissue iron concentration in the basal ganglia. We demonstrated that brain tissue iron increases throughout adolescence, with the greatest and most prolonged increases occurring in the putamen and globus pallidus. Within the globus pallidus, we also found evidence for a more protracted developmental trajectory in males relative to females. Further, we found that the developmental trajectory of tissue iron in the putamen is associated with individual differences in cognitive ability, such that individuals with diminished iron accumulation also have poorer cognitive performance in late adolescence and early adulthood.

Prior work has indicated that brain tissue iron increases most rapidly during the first two decades of life before beginning to reach a developmental asymptote (Hallgren and Sourander, 1958; Aquino et al., 2009; Wang et al., 2012; Larsen and Luna, 2015; Hect et al., 2018; Peterson et al., 2019a). The findings presented here generally support this pattern, identifying windows of significant age-related increases in $\mathrm{R} 2^{\star}$ up to ages $16-17$ in the nucleus accumbens and the caudate. However, we find that there are prolonged windows of rapid iron accumulation in the putamen and globus pallidus, where we observed significant increases in tissue iron that continued into early adulthood. Of note for future lifespan studies of the basal ganglia, the magnetic susceptibility of iron that allows for its quantification using R2* also shortens T1 and T2 relaxation time (Vymazal et al., 1995; Ogg and Steen, 1998; Stüber et al., 2014). Thus, the development of basal ganglia iron concentration may impact other MR metrics commonly used in developmental studies, such as T1-weighted imaging and diffusion weighted imaging (DWI) (Pfefferbaum et al., 2010; Peterson et al., 2019b). Given the pronounced developmental increases in brain iron concentration observed in this study, future work applying DWI or T1-based imaging metrics in developmental studies should ideally consider and control for the impact of iron.

To date, there has not been a thorough examination of sex differences in tissue iron accumulation during adolescence. In the globus pallidus, we observed an earlier developmental plateau in females, occurring $\sim$ age 22 , relative to a continued increase throughout the observed age range in males. This finding indicates a potentially longer window of iron accumulation for males during this critical developmental stage. These findings may be related to earlier work suggesting increased incidence of iron deficiency in adolescent and young adult females. The prevalence of iron deficiency in females increases from childhood to adolescence, and among nonpregnant adolescent and adult females in the United States is as high as 15\% (compared with $<3 \%$ in males) (Halterman et al., 2001; Cogswell et al., 2009; U.S. Centers for Disease Control and Prevention, 2012), and this proportion rises even higher, $>30 \%$, in female university stu-
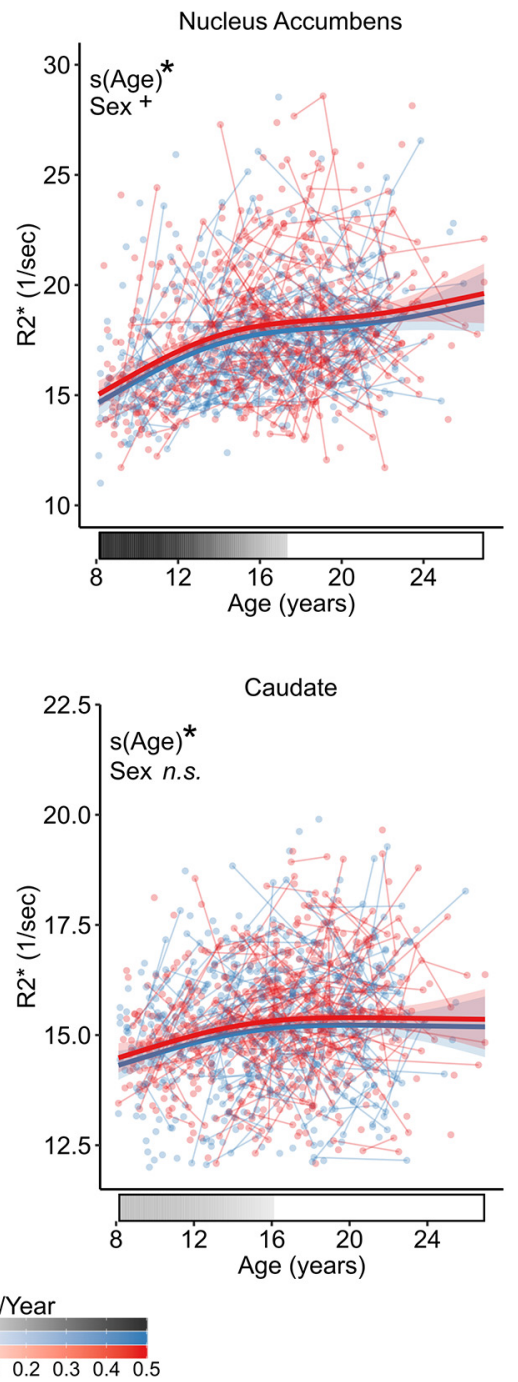

Figure 4. Development of R2* in the basal ganglia. Plots display fitted R2* trajectories from GAMM models plotted by age for Bars below the $x$-axis depict the derivative of the fitted smooth function. The filled portion of the bar indicates periods where the magnitude of derivative is significant, with the saturation of the fill representing the value of derivative in units of $\Delta R 2^{*}$ /year. ${ }^{*} p<0.05$ after Bonferroni correction; $+p<0.1$ after Bonferroni correction; n.s., not significant.

Table 3. GAMM regression table for age, sex, and interactions

\begin{tabular}{lllc}
\hline Region & Term & Statistic & $p$ \\
\hline Globus pallidus & Sex & $t=-1.3$ & 0.207 \\
& s(Age) & $F=76.7$ & $<0.0001^{*}$ \\
Nucleus accumbens & s(Age):Sex & $F=4.6$ & $0.003^{*}$ \\
Putamen & Sex & $t=2.3$ & 0.024 \\
& s(Age) & $F=41.8$ & $<0.0001^{*}$ \\
Caudate & Sex & $t=-0.9$ & 0.349 \\
& s(Age) & $F=85.8$ & $<0.0001^{*}$ \\
& Sex & $t=1.8$ & 0.068 \\
\hline
\end{tabular}

Interaction effects are reported only when the interaction is significant after Bonferroni multiple-comparison correction. Statistics are reported as $t$ statistics for parametric terms and $F$ statistics for smooth terms.

${ }^{*} p$-value is significant at the Bonferroni-adjusted $\alpha$ of 0.0125 .

dents (Worthington-Roberts et al., 1988; Houston et al., 1997; Wilcock et al., 2004; Hawk et al., 2012; Blanton, 2013). Although we did not assess dietary iron intake or serum ferritin levels in this sample, it is possible that the developmental sex differences we observe in the globus pallidus reflect developmental increases in 


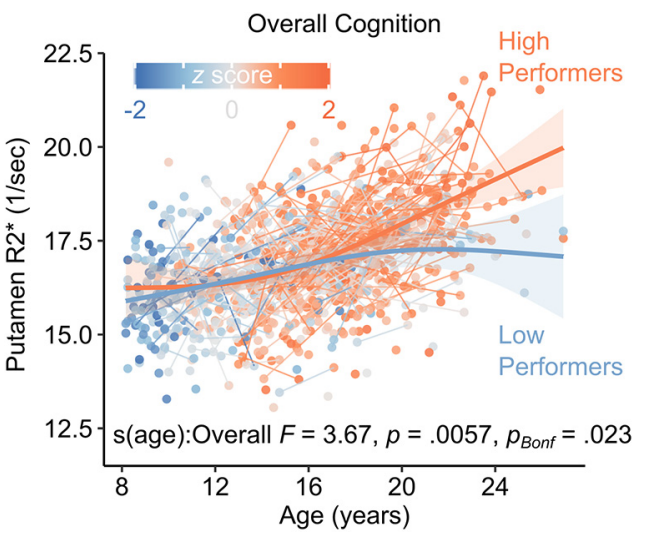

Figure 5. The development of $\mathrm{R}^{*}$ in the putamen is moderated by overall cognitive performance. To visualize the interaction between continuous variables, fitted lines are displayed that depict the relationship between age and putamen $\mathrm{R} 2 *$ for the top $5 \%$ (high performers) and bottom $95 \%$ (low performers) of the range for overall cognitive performance. Individual points represent individual scans, with connecting lines indicating scans from the same participant. The color of the points represents the overall cognitive performance $z$ score (higher values indicate more efficient performance).

Table 4. Exploratory factor analysis of longitudinal CNB tests

\begin{tabular}{llllll}
\hline Test & Social & Executive & Complex & Memory & Overall \\
\hline edi_z & 0.78 & & & & 0.77 \\
adi_z & 0.72 & & & & 0.66 \\
eid_z & 0.45 & 0.24 & & & 0.71 \\
att_z & & 0.71 & & & 0.63 \\
wm_z & & 0.64 & & & 0.61 \\
lan_z & & 0.39 & 0.35 & & 0.77 \\
nvr_z & & & 0.62 & & 0.59 \\
abf_z & & & 0.58 & & 0.53 \\
spa_z & & & 0.54 & & 0.6 \\
fmem_z & 0.23 & & & 0.56 & 0.66 \\
smem_z & & & 0.27 & 0.53 & 0.51 \\
vmem_z & & 0.33 & & 0.46 & 0.61 \\
Interfactor correlations & & & & \\
\multicolumn{1}{l}{ Social } & 1 & 0.61 & 0.68 & 0.55 & \\
Executive & 0.61 & 1 & 0.58 & 0.44 & \\
Complex & 0.68 & 0.58 & 1 & 0.41 & \\
\multicolumn{1}{l}{ Memory } & 0.55 & 0.44 & 0.41 & 1 & \\
\hline
\end{tabular}

Loadings with absolute values $<0.20$ have been removed for clarity; Tucker-Lewis Index (TLI) $=0.98$; root mean-square error of approximation (RMSEA) $=0.034 \pm 0.003$; standardized root mean-square residual $($ SRMR $)=0.01$.

the prevalence of iron deficiency in females during this developmental window.

Atypically low iron content in basal ganglia has been associated with cognitive impairment in studies of iron deficiency in early development (Beard and Connor, 2003; Beard, 2003; Carter et al., 2010; Lozoff, 2011). Here we demonstrate a similar association between reduced basal ganglia iron concentration and cognitive ability occurring within a normative developmental sample during late-adolescence and adulthood. Specifically, we find that an association between poorer overall cognitive performance and diminished iron concentration in the putamen emerges during late adolescence. When we assessed this relationship across individual subdomains of cognition, we observed that the effect was strongest in the domain of complex cognition. Adolescence has been characterized as a critical period of development of complex, higher-order cognitive functions (e.g., Larsen and Luna, 2018), and our results suggest that continued enrichment of basal ganglia iron concentration may be an important component of this developmental process. In late life, the associ- ation between brain iron concentration and cognition is reversed such that elevated brain iron is associated with neurodegeneration and impaired cognition (Bartzokis and Tishler, 2000; Ghadery et al., 2015; Zucca et al., 2017). Changing associations between brain iron content and cognitive ability during development and aging are likely driven by different underlying mechanisms. Brain iron is necessary for a myriad of critical neurobiological processes, including cellular respiration (Paul et al., 2017), myelination (Connor and Menzies, 1996; Möller et al., 2019), and neurotransmitter synthesis (Youdim et al., 1976; Lu et al., 2017). In development, a deficiency in this critical resource may impair these neurobiological processes, impairing cognition. In contrast, in aging, a breakdown of the essential homeostatic process of iron storage and metabolism can increase the risk of iron-related oxidative stress and accelerate neurodegeneration (Bartzokis et al., 2007; Núñez et al., 2012; Li and Reichmann, 2016). Longitudinal studies assessing how brain iron impacts cognitive health across the lifespan are needed.

\section{Limitations}

Despite the strengths of this study, three limitations should be noted. First, we did not collect any data on dietary iron content nor obtained serum iron status. As such, though the participants in this study were generally healthy, we cannot quantify the prevalence of iron deficiency in this sample. Thus, it is not clear whether the neurodevelopmental associations between cognitive performance and basal ganglia iron concentration we observe here are mechanistically driven by iron deficiency, as in early developmental studies (Grantham-McGregor and Ani, 2001; Beard, 2003), or if these effects are occurring within individuals with normative dietary iron content. Future work assessing brain iron concentration, peripheral iron concentration, and measures of dietary iron status should address this question directly. Additionally, as this work is focused on a normative developmental sample, and future work should investigate how developmental abnormalities of iron are associated with neuropsychiatric illness. Second, a small subset of $\mathrm{T}^{\star}$ scans were acquired with minor variations in acquisition parameters. Though we do not expect these variations to significantly impact $\mathrm{T} 2 *$ estimation, we rigorously addressed this possibility using ComBat harmonization (Johnson et al., 2007), which has been effective in harmonizing neuroimaging datasets (Fortin et al., 2017, 2018). Third, the data acquired to calculate $\mathrm{R}^{\star}{ }^{\star}$ was generated with a two-echo mGRE sequence with somewhat lower in-plane resolution $(3.75 \times 3.75$ $\mathrm{mm}$ ) than has been used in other studies (e.g., Langkammer et al., 2010; Sedlacik et al., 2014). Calculating T2* from a greater number of echo images with higher in-plane resolution can yield greater sensitivity (Anderson et al., 2001). We compensated for this limitation by using strict quality assurance procedures and having a large sample for all statistical analyses.

\section{Conclusion}

This study leverages a large longitudinal sample to characterize the normative development of tissue iron concentration across the basal ganglia. Iron accumulation follows a nonlinear developmental trajectory that begins to plateau during adolescence in the nucleus accumbens and caudate and continues to increase into the twenties in the putamen and globus pallidus. Importantly, individual differences in cognitive ability are associated with differences in putamen iron concentration during the transition from adolescence to adulthood. Together, these results highlight the transition from adolescence to adulthood as a period of dynamic maturation of tissue iron concentration in the 
A

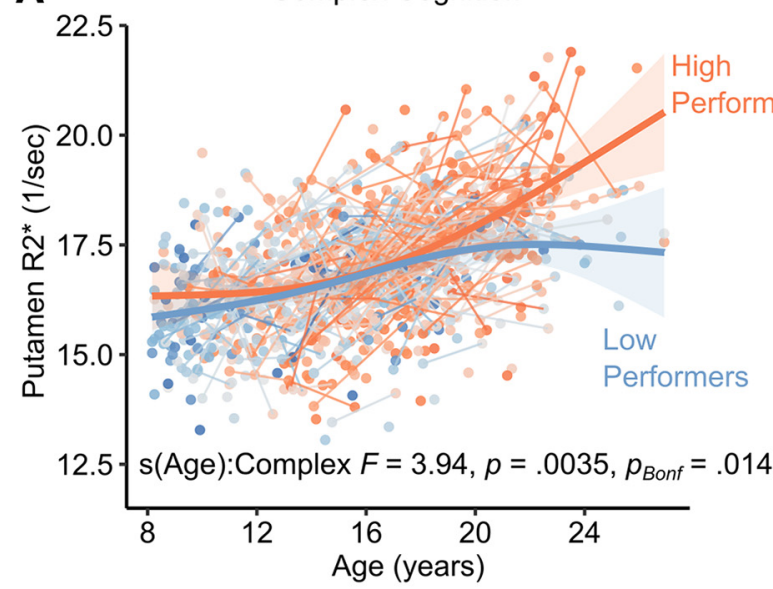

C

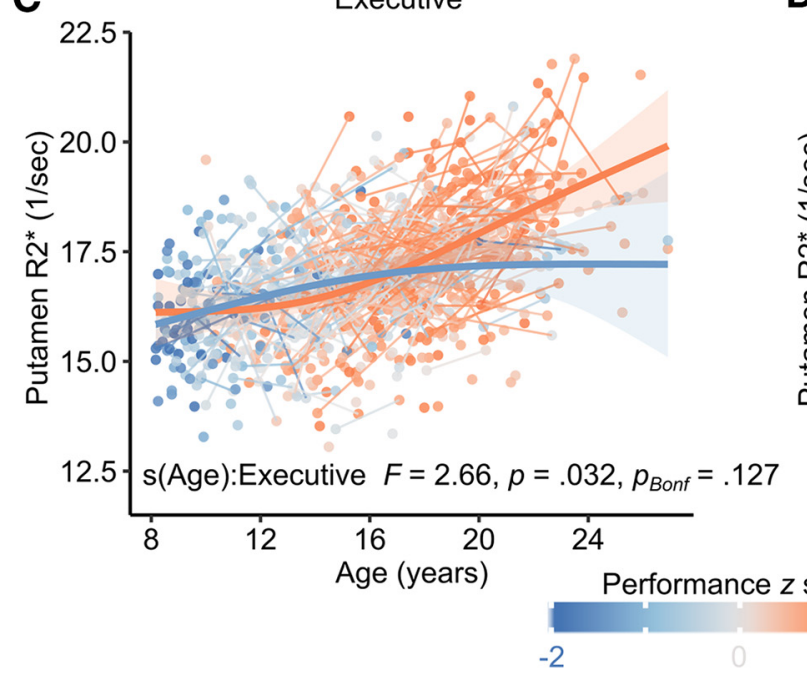

B Social Cognition

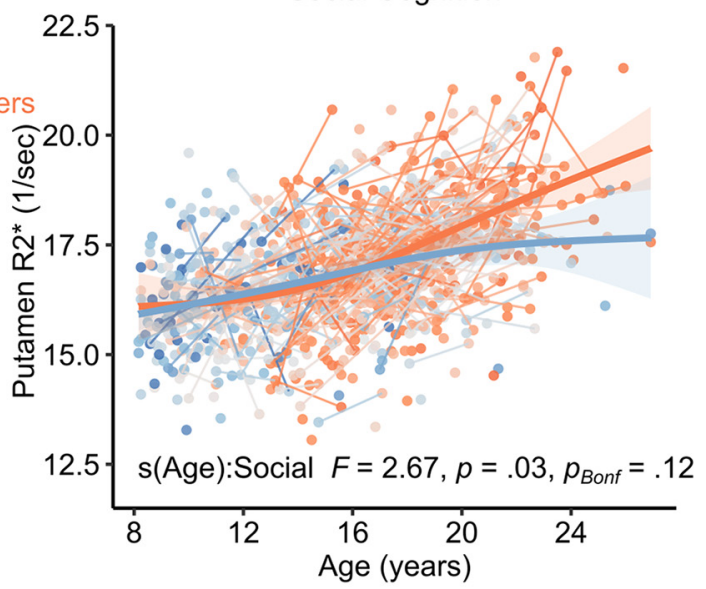

D Memory

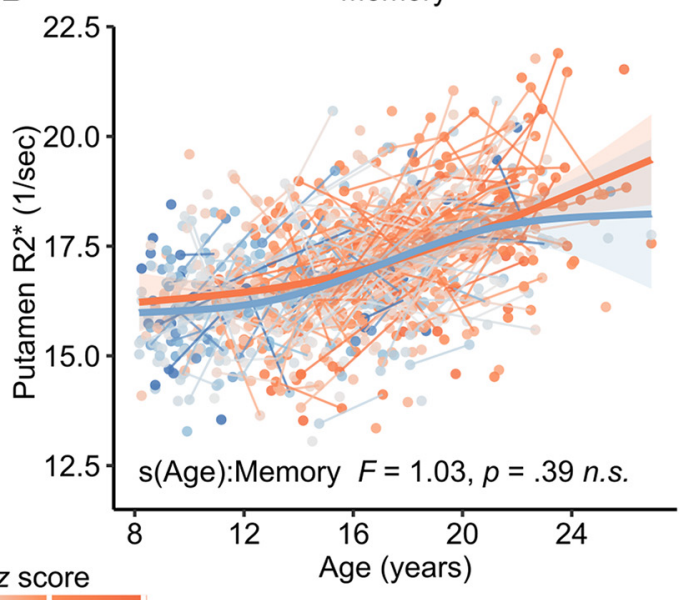

Figure 6. Cognitive moderation effects on the development of putamen $R 2^{*}$ across subdomains of cognition. Fitted values of $R 2^{*}$ are plotted as functions of age and complex cognition $(A)$, social cognition $(\boldsymbol{B})$, executive function $\left(\boldsymbol{C}\right.$, and memory $(\boldsymbol{D})$. To visualize the continuous interactions, fitted lines are displayed that depict the relationship between age and putamen $\mathrm{R} 2^{*}$ for the top $5 \%$ (High performers) and bottom 95\% (Low performers) of the range for each cognitive measure. Individual points represent individual scans, with connecting lines indicating scans from the same participant. The color of the points represents the cognitive $z$ score (higher values indicating more efficient performance).

basal ganglia that may affect individual variability in complex cognitive performance in adulthood. Importantly, as iron supplementation has been found to improve cognitive performance in iron deficient adolescents (Bruner et al., 1996; Blanton, 2013), it is possible that efforts to enhance brain iron concentration through dietary iron supplementation may have beneficial effects on cognitive development during this critical period of development. Studies that prospectively evaluate the longitudinal impact of iron supplementation on cognition and brain development are necessary.

\section{References}

Adamo DE, Daugherty AM, Raz N (2014) Grasp force matching and brain iron content estimated in vivo in older women. Brain Imaging Behav 8:579-587.

Anderson LJ, Holden S, Davis B, Prescott E, Charrier CC, Bunce NH, Firmin DN, Wonke B, Porter J, Walker JM, Pennell DJ (2001) Cardiovascular $\mathrm{T} 2$-star $\left(\mathrm{T} 2^{*}\right)$ magnetic resonance for the early diagnosis of myocardial iron overload. Eur Heart J 22:2171-2179.

Aquino D, Bizzi A, Grisoli M, Garavaglia B, Bruzzone MG, Nardocci N, Savoiardo M, Chiapparini L (2009) Age-related iron deposition in the basal ganglia: quantitative analysis in healthy subjects. Radiology 252: $165-172$.

Avants BB, Epstein CL, Grossman M, Gee JC (2008) Symmetric diffeomor- phic image registration with cross-correlation: evaluating automated labeling of elderly and neurodegenerative brain. Med Image Anal 12:26-41.

Avants BB, Tustison NJ, Song G, Cook PA, Klein A, Gee JC (2011a) A reproducible evaluation of ANTs similarity metric performance in brain image registration. Neuroimage 54:2033-2044.

Avants BB, Tustison NJ, Wu J, Cook PA, Gee JC (2011b) An open source multivariate framework for $\mathrm{n}$-tissue segmentation with evaluation on public data. Neuroinformatics 9:381-400.

Bartzokis G, Tishler TA (2000) MRI evaluation of basal ganglia ferritin iron and neurotoxicity in Alzheimer's and Huntingon's disease. Cell Mol Biol 46:821-833.

Bartzokis G, Beckson M, Hance DB, Marx P, Foster JA, Marder SR (1997) MR evaluation of age-related increase of brain iron in young adult and older normal males. Magn Reson Imaging 15:29-35.

Bartzokis G, Cummings J, Perlman S, Hance DB, Mintz J (1999) Increased basal ganglia iron levels in Huntington disease. Arch Neurol 56:569-574.

Bartzokis G, Sultzer D, Cummings J, Holt LE, Hance DB, Henderson VW, Mintz J (2000) In vivo evaluation of brain iron in Alzheimer disease using magnetic resonance imaging. Arch Gen Psychiatry 57:47-53.

Bartzokis G, Tishler TA, Lu PH, Villablanca P, Altshuler LL, Carter M, Huang D, Edwards N, Mintz J (2007) Brain ferritin iron may influence age- and gender-related risks of neurodegeneration. Neurobiol Aging 28:414-423.

Beard J (2003) Iron deficiency alters brain development and functioning. J Nutr 133:1468S-1472S. 
Beard JL, Connor JR (2003) Iron status and neural functioning. Annu Rev Nutr 23:41-58.

Blanton C (2013) Improvements in iron status and cognitive function in young women consuming beef or non-beef lunches. Nutrients 6:90-110.

Brass SD, Chen NK, Mulkern RV, Bakshi R (2006) Magnetic resonance imaging of iron deposition in neurological disorders. Top Magn Reson Imaging 17:31-40.

Bruner AB, Joffe A, Duggan AK, Casella JF, Brandt J (1996) Randomised study of cognitive effects of iron supplementation in non-anaemic irondeficient adolescent girls. Lancet 348:992-996.

Burdo JR, Connor JR (2003) Brain iron uptake and homeostatic mechanisms: an overview. Biometals 16:63-75.

Carter RC, Jacobson JL, Burden MJ, Armony-Sivan R, Dodge NC, Angelilli ML, Lozoff B, Jacobson SW (2010) Iron deficiency anemia and cognitive function in infancy. Pediatrics 126:e427-e434.

Cogswell ME, Looker AC, Pfeiffer CM, Cook JD, Lacher DA, Beard JL, Lynch SR, Grummer-Strawn LM (2009) Assessment of iron deficiency in US preschool children and nonpregnant females of childbearing age: national health and nutrition examination survey 2003-2006. Am J Clin Nutr 89:1334-1342.

Connor JR, Menzies SL (1996) Relationship of iron to oligodendrocytes and myelination. Glia 17:83-93.

Daugherty AM, Raz N (2015) Appraising the role of iron in brain aging and cognition: promises and limitations of MRI methods. Neuropsychol Rev 25:272-287.

Erikson KM, Jones BC, Beard JL (2000) Iron deficiency alters dopamine transporter functioning in rat striatum. J Nutr 130:2831-2837.

Erikson KM, Jones BC, Hess EJ, Zhang Q, Beard JL (2001) Iron deficiency decreases dopamine D1 and D2 receptors in rat brain. Pharmacol Biochem Behav 69:409-418.

Fortin JP, Parker D, Tunç B, Watanabe T, Elliott MA, Ruparel K, Roalf DR, Satterthwaite TD, Gur RC, Gur RE, Schultz RT, Verma R, Shinohara RT (2017) Harmonization of multi-site diffusion tensor imaging data. Neuroimage 161:149-170.

Fortin JP, Cullen N, Sheline YI, Taylor WD, Aselcioglu I, Cook PA, Adams P, Cooper C, Fava M, McGrath PJ, McInnis M, Phillips ML, Trivedi MH, Weissman MM, Shinohara RT (2018) Harmonization of cortical thickness measurements across scanners and sites. Neuroimage 167:104-120.

Ghadery C, Pirpamer L, Hofer E, Langkammer C, Petrovic K, Loitfelder M, Schwingenschuh P, Seiler S, Duering M, Jouvent E, Schmidt H, Fazekas F, Mangin JF, Chabriat H, Dichgans M, Ropele S, Schmidt R (2015) R2* mapping for brain iron: associations with cognition in normal aging. Neurobiol Aging 36:925-932.

Grantham-McGregor S, Ani C (2001) A review of studies on the effect of iron deficiency on cognitive development in children. J Nutr 131: 649S-668S.

Griffiths PD, Crossman AR (1993) Distribution of iron in the basal ganglia and neocortex in postmortem tissue in Parkinson's disease and Alzheimer's disease. Dementia 4:61-65.

Gur RC, Richard J, Hughett P, Calkins ME, Macy L, Bilker WB, Brensinger C, Gur RE (2010) A cognitive neuroscience-based computerized battery for efficient measurement of individual differences: standardization and initial construct validation. J Neurosci Methods 187:254-262.

Gur RC, Richard J, Calkins ME, Chiavacci R, Hansen JA, Bilker WB, Loughead J, Connolly JJ, Qiu H, Mentch FD, Abou-Sleiman PM, Hakonarson H, Gur RE (2012) Age group and sex differences in performance on a computerized neurocognitive battery in children age 8-21. Neuropsychology 26:251-265.

Gur RE, Gur RC (2016) Sex differences in brain and behavior in adolescence: findings from the Philadelphia neurodevelopmental cohort. Neurosci Biobehav Rev 70:159-170.

Haacke EM, Miao Y, Liu M, Habib CA, Katkuri Y, Liu T, Yang Z, Lang Z, Hu J, Wu J (2010) Correlation of change in $\mathrm{R} 2^{*}$ and phase with putative iron content in deep gray matter of healthy adults. J Magn Reson Imaging 32:561-576.

Haber SN, Knutson B (2010) The reward circuit: linking primate anatomy and human imaging. Neuropsychopharmacology 35:4-26.

Halekoh U, Højsgaard S (2014) A Kenward-Roger approximation and parametric bootstrap methods for tests in linear mixed models: the R package pbkrtest. Journal of Statistical Software 59:1-32.

Hallgren B, Sourander P (1958) The effect of age on the non-haemin iron in the human brain. J Neurochem 3:41-51.
Halterman JS, Kaczorowski JM, Aligne CA, Auinger P, Szilagyi PG (2001) Iron deficiency and cognitive achievement among school-aged children and adolescents in the united states. Pediatrics 107:1381-1386.

Hawk SN, Englehardt KG, Small C (2012) Risks of iron deficiency among vegetarian college women. Health 4:113-119.

Hect JL, Daugherty AM, Hermez KM, Thomason ME (2018) Developmental variation in regional brain iron and its relation to cognitive functions in childhood. Dev Cogn Neurosci 34:18-26.

Houston MS, Summers SL, Soltesz KS (1997) Lifestyle and dietary practices influencing iron status in university women. Nutr Res 17:9-22.

Jellen LC, Lu L, Wang X, Unger EL, Earley CJ, Allen RP, Williams RW, Jones BC (2013) Iron deficiency alters expression of dopamine-related genes in the ventral midbrain in mice. Neuroscience 252:13-23.

Jirsaraie RJ, Kaczkurkin AN, Rush S, Piiwia K, Adebimpe A, Bassett DS, Bourque J, Calkins ME, Cieslak M, Ciric R, Cook PA, Davila D, Elliott MA, Leibenluft E, Murtha K, Roalf DR, Rosen AFG, Ruparel K, Shinohara RT, Sotiras A, et al. (2019) Accelerated cortical thinning within structural brain networks is associated with irritability in youth. Neuropsychopharmacology 44:2254-2262.

Johnson WE, Li C, Rabinovic A (2007) Adjusting batch effects in microarray expression data using empirical Bayes methods. Biostatistics 8:118-127.

Kell DB (2010) Towards a unifying, systems biology understanding of largescale cellular death and destruction caused by poorly liganded iron: Parkinson's, Huntington's, Alzheimer's, prions, bactericides, chemical toxicology and others as examples. Arch Toxicol 84:825-889.

Klein A, Andersson J, Ardekani BA, Ashburner J, Avants B, Chiang MC, Christensen GE, Collins DL, Gee J, Hellier P, Song JH, Jenkinson M, Lepage C, Rueckert D, Thompson P, Vercauteren T, Woods RP, Mann JJ, Parsey RV (2009) Evaluation of 14 nonlinear deformation algorithms applied to human brain MRI registration. Neuroimage 46:786-802.

Kuhn DM, Ruskin B, Lovenberg W (1980) Tryptophan hydroxylase. the role of oxygen, iron, and sulfhydryl groups as determinants of stability and catalytic activity. J Biol Chem 255:4137-4143.

Langkammer C, Krebs N, Goessler W, Scheurer E, Ebner F, Yen K, Fazekas F, Ropele S (2010) Quantitative MR imaging of brain iron: a postmortem validation study. Radiology 257:455-462.

Larsen B, Luna B (2015) In vivo evidence of neurophysiological maturation of the human adolescent striatum. Dev Cogn Neurosci 12:74-85.

Larsen B, Luna B (2018) Adolescence as a neurobiological critical period for the development of higher-order cognition. Neurosci Biobehav Rev 94: 179-195.

Li K, Reichmann H (2016) Role of iron in neurodegenerative diseases. J Neural Transm 123:389-399.

Lozoff B (2011) Early iron deficiency has brain and behavior effects consistent with dopaminergic dysfunction. J Nutr 141:740S-746S.

Lozoff B, Georgieff MK (2006) Iron deficiency and brain development. Semin Pediatr Neurol 13:158-165.

Lu H, Chen J, Huang H, Zhou M, Zhu Q, Yao SQ, Chai Z, Hu Y (2017) Iron modulates the activity of monoamine oxidase B in SH-SY5Y cells. Biometals 30:599-607.

Lv Z, Jiang H, Xu H, Song N, Xie J (2011) Increased iron levels correlate with the selective nigral dopaminergic neuron degeneration in Parkinson's disease. J Neural Transm 118:361-369.

McCann JC, Ames BN (2007) An overview of evidence for a causal relation between iron deficiency during development and deficits in cognitive or behavioral function. Am J Clin Nutr 85:931-945.

Mills E, Dong XP, Wang F, Xu H (2010) Mechanisms of brain iron transport: insight into neurodegeneration and CNS disorders. Future Med Chem 2:51-64.

Möller HE, Bossoni L, Connor JR, Crichton RR, Does MD, Ward RJ, Zecca L, Zucca FA, Ronen I (2019) Iron, myelin, and the brain: neuroimaging meets neurobiology. Trends Neurosci 42:384-401.

Moore TM, Reise SP, Gur RE, Hakonarson H, Gur RC (2015) Psychometric properties of the Penn computerized neurocognitive battery. Neuropsychology 29:235-246.

Moos T (2002) Brain iron homeostasis. Dan Med Bull 49:279-301.

Núñez MT, Urrutia P, Mena N, Aguirre P, Tapia V, Salazar J (2012) Iron toxicity in neurodegeneration. Biometals 25:761-776.

Ogg RJ, Steen RG (1998) Age-related changes in brain T1 are correlated with iron concentration. Magn Reson Med 40:749-753.

Paul BT, Manz DH, Torti FM, Torti SV (2017) Mitochondria and iron: current questions. Expert Rev Hematol 10:65-79. 
Penke L, Valdés Hernandéz MC, Maniega SM, Gow AJ, Murray C, Starr JM, Bastin ME, Deary IJ, Wardlaw JM (2012) Brain iron deposits are associated with general cognitive ability and cognitive aging. Neurobiol Aging 33:510-517.e2.

Péran P, Cherubini A, Luccichenti G, Hagberg G, Démonet JF, Rascol O, Celsis P, Caltagirone C, Spalletta G, Sabatini U (2009) Volume and iron content in basal ganglia and thalamus. Hum Brain Mapp 30:2667-2675.

Peterson ET, Kwon D, Luna B, Larsen B, Prouty D, De Bellis MD, Voyvodic J, Liu C, Li W, Pohl KM, Sullivan EV, Pfefferbaum A (2019a) Distribution of brain iron accrual in adolescence: evidence from cross-sectional and longitudinal analysis. Hum Brain Mapp 40:1480-1495.

Peterson ET, Kwon D, Luna B, Larsen B, Prouty D, De Bellis MD, Voyvodic J, Liu C, Li W, Pohl KM, Sullivan EV, Pfefferbaum A (2019b) Distribution of brain iron accrual in adolescence: evidence from cross-sectional and longitudinal analysis. Hum Brain Mapp 40:1480-1495.

Pfefferbaum A, Adalsteinsson E, Rohlfing T, Sullivan EV (2010) Diffusion tensor imaging of deep gray matter brain structures: effects of age and iron concentration. Neurobiol Aging 31:482-493.

Pujol J, Junqué C, Vendrell P, Grau JM, Martí-Vilalta JL, Olivé C, Gili J (1992) Biological significance of iron-related magnetic resonance imaging changes in the brain. Arch Neurol 49:711-717.

Ramsey AJ, Hillas PJ, Fitzpatrick PF (1996) Characterization of the active site iron in tyrosine hydroxylase redox states of the iron. J Biol Chem 271:24395-24400.

Reise SP, Cook KF, Moore TM (2015) Evaluating the impact of multidimensionality on unidimensional item response theory model parameters. In: Handbook of item response theory modeling: Applications to typical performance assessment, pp 13-40 Multivariate applications series. New York, NY, US: Routledge/Taylor \& Francis Group.

Roalf DR, Garza AG de la, Rosen A, Calkins ME, Moore TM, Quarmley M, Ruparel K, Xia CH, Rupert PE, Satterthwaite TD, Shinohara RT, Elliott MA, Gur RC, Gur RE (2019) Alterations in white matter microstructure in individuals at persistent risk for psychosis. Mol Psychiatry. Advance online publication. Retrieved February 5, 2019. doi: 10.1038/s41380-019-0360-1.

Sedlacik J, Boelmans K, Löbel U, Holst B, Siemonsen S, Fiehler J (2014) Reversible, irreversible and effective transverse relaxation rates in normal aging brain at 3 T. Neuroimage 84:1032-1041.

Shafir T, Angulo-Barroso R, Jing Y, Angelilli ML, Jacobson SW, Lozoff B (2008) Iron deficiency and infant motor development. Early Hum Dev $84: 479-485$.

Simpson GL (2018) Modelling palaeoecological time series using generalised additive models. In: Frontiers in Ecology and Evolution. Available at: https://www.frontiersin.org/articles/10.3389/fevo.2018.00149/full. Accessed September 9, 2019.

Stüber C, Morawski M, Schäfer A, Labadie C, Wähnert M, Leuze C, Streicher M, Barapatre N, Reimann K, Geyer S, Spemann D, Turner R (2014)
Myelin and iron concentration in the human brain: a quantitative study of MRI contrast. Neuroimage 93:95-106.

Thomas LO, Boyko OB, Anthony DC, Burger PC (1993) MR detection of brain iron. AJNR Am J Neuroradiol 14:1043-1048.

Todorich B, Pasquini JM, Garcia CI, Paez PM, Connor JR (2009) Oligodendrocytes and myelination: the role of iron. Glia 57:467-478.

Tustison NJ, Avants BB, Cook PA, Zheng Y, Egan A, Yushkevich PA, Gee JC (2010) N4ITK: improved N3 bias correction. IEEE Trans Med Imaging 29:1310-1320.

U.S. Centers for Disease Control and Prevention (2012) Second national report on biochemical indicators of diet and nutrition in the U.S. population. Atlanta: National Center for Environmental Health.

Vymazal J, Hajek M, Patronas N, Giedd JN, Bulte JW, Baumgarner C, Tran V, Brooks RA (1995) The quantitative relation between T1-weighted and T2-weighted MRI of normal gray matter and iron concentration. J Magn Reson Imaging 5:554-560.

Wang H, Suh JW, Das SR, Pluta JB, Craige C, Yushkevich PA (2013) Multiatlas segmentation with joint label fusion. IEEE Trans Pattern Anal Mach Intell 35:611-623.

Wang J, Shaffer ML, Eslinger PJ, Sun X, Weitekamp CW, Patel MM, Dossick D, Gill DJ, Connor JR, Yang QX (2012) Maturational and aging effects on human brain apparent transverse relaxation. PLoS One 7:e31907.

Ward RJ, Zucca FA, Duyn JH, Crichton RR, Zecca L (2014) The role of iron in brain ageing and neurodegenerative disorders. Lancet Neurol 13:1045-1060.

Wilcock C, Wengreen H, Schvaneveldt NB (2004) Iron deficiency in youngadult women attending Utah State University. Journal of the Academy of Nutrition and Dietetics 104:27.

Wood SN (2004) Stable and efficient multiple smoothing parameter estimation for generalized additive models. Journal of the American Statistical Association 99:673-686.

Wood SN (2017) Generalized additive models: an introduction with R, Ed 2. New York: Chapman and Hall/CRC. Available at: https://www.taylorfrancis.com/books/9781315370279. Accessed August 8, 2019.

Worthington-Roberts BS, Breskin MW, Monsen ER (1988) Iron status of premenopausal women in a university community and its relationship to habitual dietary sources of protein. Am J Clin Nutr 47:275-279.

Youdim MBH (2018) Monoamine oxidase inhibitors, and iron chelators in depressive illness and neurodegenerative diseases. J Neural Transm 125: 1719-1733.

Youdim MB, Grahame-Smith DG, Woods HF (1976) Some properties of human platelet monoamine oxidase in iron-deficiency anaemia. Clin Sci Mol Med 50:479-485.

Zucca FA, Segura-Aguilar J, Ferrari E, Muñoz P, Paris I, Sulzer D, Sarna T, Casella L, Zecca L (2017) Interactions of iron, dopamine and neuromelanin pathways in brain aging and Parkinson's disease. Prog Neurobiol 155:96-119. 